\title{
Application of Non-certificate Multi-level Proxy Digital Signature Scheme in Online Banking Transaction
}

\author{
Lin $\mathrm{Zhu}^{1}$ \\ ${ }^{1}$ School of Business, Sias International University, Henan XinZheng, China \\ E-mail:15345395@qq.com
}

\begin{abstract}
In order to resist to active attacks, Chen Jiaqi has proposed an authenticated noncertificate three-party key negotiation protocol in 2010, but this key protocol cannot resist to passive attacks or meet the requirements for some security attributes mentioned in his paper. In order to solve the above problems, He De-biao has proposed a new authenticated non-certificate three-party key negotiation protocol on the basis of noncertificate two-party key negotiation protocol. The results of the security analysis and the efficiency analysis of the new protocol show that the new protocol can meet the security requirements of the authenticated non-certificate three-party key negotiation protocol. Compared with the protocol proposed by Chen Jiaqi, the new protocol has less bilinear pairings and higher calculation efficiency.
\end{abstract}

Keywords: Non-certificate; Authenticated; Key negotiation; Bilinear pairings

\section{Introduction}

Along with the popularization of the wireless network, the quantity of the information transmitted by the wireless network is rapidly increased. Meanwhile, stricter and stricter security requirements are proposed for the information transmission. As an important protocol for ensuring the secure communication, the key negotiation protocol can guarantee two or more communication parties to jointly find a shared session key by which the communication parties can have secure communication in the channels. As the most universal communication mode for electronic conference, the three-party key negotiation has important application value, thus to be deeply researched by many scholars. Identity based authentication key negotiation protocol [10] for different PKG fields is also researched in some literatures. Although Chen Jiaqi has proposed an authenticated non-certificate three-party key negotiation protocol in 2010, yet this protocol also has security defect. In order to eliminate the security defect existing in the protocol proposed by Chen Jiachi, He Debiao has proposed a new authenticated noncertificate three-party key negotiation protocol on the basis of the non-certificate twoparty key negotiation protocol [12].

\section{Authenticated Non-certificate Three-party Key Negotiation Protocol}

(1) Generation of system parameters: $G 1$ is the cyclic additive group on the elliptic curve and $G 2$ is the cyclic multiplicative group, their orders are both as $q$, and $P$ is the generator of $G 1$. A bilinear mapping $e$ is defined as $e: G_{1} \times G_{1} \rightarrow G_{2}$, and the two one-way Hash functions are defined as $H_{1}:\{0,1\}^{*} \rightarrow G_{1}$ and $H_{2}: G_{2} \times G_{1} \times G_{1} \times G_{1} \rightarrow Z_{q}^{*} . \quad s \in Z_{q}^{*}$ is randomly selected in the key generation center (KGC) as the master key of the system, and the public key of the system is calculated as $P_{p u b}=s P$, and s is saved, and the system parameter is issued as ${ }^{\text {params }}=\left\{G_{1}, G_{2}, e, q, P, P_{p u}, H_{1}, H_{2}\right\}$. 
(2) Extraction of partial private key: user $A$ provides identity information $I D A$ to $\mathrm{KGC}$, and after authenticating IDA, KGC inputs parameter ${ }^{\text {params }}$, master key $s$ and IDA in the system, calculates $Q A$ and partial private key $D A$, and sends $Q A$ and $D A$ to $A$ through secure channel, wherein $Q_{A}=H_{1}\left(I D_{A}\right)$ and $D_{A}=s Q_{A}$. A can verify the authenticity of $D A$ through equation $^{e\left(D_{A}, P\right)=e\left(Q_{A}, P_{p u b}\right)}$.

(3) Extraction of secret value: user $A$ selects ${ }_{A} \in Z_{q}^{*}$ as the long-term secret value.

(4) Generation of private key: this algorithm is executed at the client of $A, A$ inputs parameter ${ }^{\text {params }}$, partial $D A$ and secret value ${ }^{x_{A}}$, and the client outputs private key $S A$, wherein $S_{A}=\left\langle D_{A}, x_{A}\right\rangle$.

(5) Generation of public key: system parameter ${ }^{\text {params }}$ and the secret value ${ }^{x_{A}}$ of user $A$ are input to output public key $P A$, wherein $P_{A}=x_{A} P$.

(6) Key negotiation of single KGC: users $A, B$ and $C$ respectively provide their identity information $I D A, I D B$ and $I D C$ to $\mathrm{KGC}$, and then KGC returns partial private keys $D A$, $D B$ and $D C$ thereto through the secure channel, wherein $D_{i}=s Q_{i}=s H_{1}\left(I D_{i}\right)$ and $s$ is the master key of KGC. Each participant user needs to generate a secret value ${ }^{x_{i} \in Z_{q}^{*}}$ not known by $K G C$ and then combine partial private key $D i$ to generate private key $S_{i}=\left\langle D_{i}, x_{i}\right\rangle$, wherein the public key of the users is $P_{i}=x_{i} P$ and the short-term key is $S_{i}^{\prime}=D_{i}+x_{i} Q_{i}=\left(s+x_{i}\right) Q_{i}$.

\section{Attack to Non-certificate Three-party Key Negotiation Protocol}

\subsection{Passive Attack}

According to the protocol proposed by Chen Jiaqi, the three parties can finally obtain a shared key ${ }^{K=e}\left(Q_{A}, P\right)^{\left(s+s+x_{A}\right)} \cdot e\left(Q_{B}, P\right)^{b\left(s+x_{B}\right)} \cdot e\left(Q_{C}, P\right)^{\left(s+x_{C}\right)}$ which can be transformed as follows:

$$
\begin{aligned}
K & =e\left(Q_{A}, P\right)^{a\left(s+x_{A}\right)} \cdot e\left(Q_{B}, P\right)^{b\left(s+x_{B}\right)} \cdot e\left(Q_{C}, P\right)^{\left(s+x_{C}\right)} \\
& =e\left(a Q_{A},\left(s+x_{A}\right) P\right) \cdot e\left(b Q_{B},\left(s+x_{B}\right) P\right) \cdot e\left(c Q_{C},\left(s+x_{C}\right) P\right) \\
& =e\left(T_{A}, P_{p u b}+P_{A}\right) \cdot e\left(T_{B}, P_{p u b}+P_{B}\right) \cdot e\left(T_{C}, P_{p u b}+P_{C}\right)
\end{aligned}
$$

According to the above results, $P_{p u b}, P_{A}, P_{B}, P_{C}$ are public information. If there is any passive opponent during the communication, this passive opponent can calculate the shared key $K$ when capturing messages $T A, T B$ and $T C$ during the three-party communication, and accordingly calculate the final session key of this protocol. Moreover, the passive opponent can decrypt any secret information encrypted by the three parties through the session key.

\subsection{Dissatisfaction of Forward Security}

The following condition is assumed for the key negotiation process: if the opponent knows the private keys $S K_{A}=\left(x_{A}, D_{A}\right), S K_{B}=\left(x_{B}, D_{B}\right), S K_{C}=\left(x_{C}, D_{C}\right)$ of users A, B and C, then the opponent can control the whole communication channel. Accordingly, the opponent can obtain TA, TB and TC, and due to the public information $P_{p u b}, P_{A}, P_{B}, P_{C}$, the opponent can finally calculate the previous shared key $\mathrm{K}$ and the previous session key. In conclusion, the protocol proposed by Chen Jiachi cannot meet the forward security requirement.

\subsection{Dissatisfaction of Known Session Security}

If the opponent is assumed to know the session key $K l$ of users $A, B$ and $C$ at a certain moment, since the opponent can control the whole communication channel, the opponent can obtain $T A, T B$ and $T C$. Moreover, due to the public information $P_{p u b}, P_{A}, P_{B}, P_{C}$, the 
opponent can calculate the shared key $K 2$ of users A, B and C and accordingly calculate the session key. Obviously, the protocol proposed by Chen Jiaqi cannot meet the known session security requirement.

\subsection{Irresistibility to Key Exposure Impersonation Attack}

The opponent is assumed to know partial private key $D A$ of user A. Firstly, we will discuss the first kind of opponents, namely external opponent; he can replace the public key of the users, but does not know the partial private keys of the users; he can randomly select ${ }^{x_{B}^{\prime}, b^{\prime} \in Z_{q}^{*}}$, calculate $P_{B}^{P^{\prime}}=x_{B}^{\prime} P, T_{B}^{\prime}=b^{\prime} Q_{B}$ and send ${ }^{\left(P_{B}^{\prime}, T_{B}^{\prime}\right)}$ respectively to $A$ and $C$; finally, $A$ can calculate $\quad K_{A}=e\left(S_{A}^{\prime}, P\right)^{a} \cdot e\left(T_{B}^{\prime}, P_{p u b}+P_{B}^{\prime}\right) \cdot e\left(T_{C}, P_{p u b}+P_{C}\right) \quad ; \quad$ due to $e\left(S_{A}^{\prime}, P\right)^{a}=e\left(\left(s+x_{A}\right) Q_{A}, P\right)^{a}=e\left(a Q_{A},\left(s+x_{A}\right) P\right)=e\left(T_{A}, P_{\text {nut }}+P_{A}\right)$, the opponent can finally calculate the shared key $K A$ to pose as B for A. Then, we will discuss the second kind of opponents, namely the internal opponent; he knows the master key but cannot replace the public key of the users; he can randomly select ${ }^{b^{\prime} \in Z_{q}^{*}}$, calculate ${ }^{T_{B}^{\prime}}=b^{\prime} Q_{B}$ and send ${ }^{T_{B}^{\prime}}$ respectively to $A$ and $C$; finally, $A$ can calculate the shared key $K_{A}=e\left(S_{A}^{\prime}, P\right)^{a} \cdot e\left(T_{B}^{\prime}, P_{p u b}+P_{B}\right) \cdot e\left(T_{C}, P_{p u b}+P_{C}\right)$; similarly, the opponent can also calculate the shared key $K A$ to pose as $B$ for $A$. According to the analysis of the above two kinds of opponents, the protocol proposed by Chen Jiaqi cannot resist to key exposure impersonation attacks.

\section{New Authenticated Non-certificate Three-party Key Negotiation Protocol}

\subsection{Non-certificate Three-party Key Negotiation Protocol for Single KGC}

(1) Generation of system parameters: the security parameter is $k \in Z$, and the algorithm operation is as follows:

(1) Input parameter $k$ and run the parameter generator to obtain prime number, wherein $G 1$ and $G 2$ are two cyclic groups, $G 1$ is $q$-order cyclic additive group, $G 2$ is $q$ order cyclic multiplicative group, and $P$ is the generator of $G 1$.

(2) Randomly select master key ${ }^{s \in Z_{q}^{*}}$ and calculate master public key ${ }^{P_{u b}}=s P$.

(3) Select the security Hash functions in the cryptosystem: $H_{1}:\{0,1\}^{*} \times G_{1} \rightarrow Z_{q}^{*}$ and $H_{2}: G_{2} \times G_{2} \times G_{1} \times G_{1} \rightarrow\{0,1\}^{k}$; finally, secretly save master key $s$ of PKG and disclose system parameters $\left\{q, G_{1}, G_{2}, e, P, P_{p u b}, H_{1}, H_{2}\right\}$.

(2) Extraction of partial private key: after user identity ${ }^{I D_{i}} \in\{0,1\}^{*}$ is provided, KGC firstly randomly selects ${ }^{r_{i} \in Z_{q}^{*}}$, calculates $R_{i}=r_{i} P, h=H_{1}\left(I D_{i}, R_{i}\right), D_{i}=r_{i}+h_{i} s$, sets partial private key $(D i, R i))$ of the user, and then secretly sends partial private key $(D i, R i)$ to user IDi.

After receiving partial private key ( $\mathrm{Di}, \mathrm{Ri})$, the user verifies the validity thereof: if $D_{i} \cdot P=R_{i}+h_{i} \cdot P_{p u b}$ is true, then the partial private key is deemed to be valid; or else, it is deemed to be invalid.

(3) Secret value setting: user IDi randomly selects ${ }^{x_{i}} \in Z_{q}^{*}$, and calculates ${ }_{i}=x_{i} P$, wherein $x i$ is set as his secret value.

(4) Private key setting: the private key of user IDi is set as $s k_{i}=\left(x_{i}, D_{i}\right)$.

(5) Public key setting: the public key of user IDi is set as ${ }^{p k_{i}}=P_{i}$.

(6) The key negotiation process is as follows: 
For entity A with identity as IDA, the private key and the public key thereof are respectively set as $s k_{A}=\left(x_{A}, D_{A}\right)$ and $p k_{A}=P_{A}$; for entity B with the identity as $I D B$, the private key and the public key thereof are respectively set as ${ }^{s k_{B}}=\left(x_{B}, D_{B}\right)$ and ${ }^{p k_{B}}=P_{B}$; for entity $C$ with the identity as $I D C$, the private key and the public key thereof are respectively set as $s k_{C}=\left(x_{c}, D_{c}\right)$ and $p k_{c}=P_{C}$. They intend to establish a shared session key, and the communication process is as follows:

(1) $A, B$ and $C$ respectively randomly select secret values ${ }^{a, b, c \in Z_{q}^{*}}$ and calculate $T_{A}=a P, T_{B}=b P, T_{C}=c P$.

(2) $A$ sends $\left\langle I D_{A}, T_{A}, P_{A}, R_{A}\right\rangle$ to $B$ and $C$; then $B$ sends $\left\langle I D_{B}, T_{B}, P_{B}, R_{B}\right\rangle$ to $A$ and $C$.

(3) After receiving the messages from $A$ and $B, C$ calculates as follows:

$$
\begin{aligned}
& K_{C_{1}}=e\left(R_{A}+H_{1}\left(I D_{A}, R_{A}\right) P_{\text {pub }}+T_{A}, R_{B}+H_{1}\left(I_{B}, R_{B}\right) P_{m b b}+T_{B}\right)^{c+D_{C}} \\
& K_{C_{2}}=e\left(P_{A}, P_{B}\right)^{*}
\end{aligned}
$$

$C$ sends $\left\langle I D_{c}, T_{C}, P_{C}, R_{C}, M A C_{\kappa_{\mathrm{q}} / 1 / \kappa_{c}}\left(I D_{c}, T_{C}, P_{C}, R_{C}\right)\right\rangle$ to $A$ and $B$, wherein $M A C$ is the message authentication code of the user for ensuring data integrity.

(4) After receiving the message from $C, A$ and $B$ respectively calculate ${ }^{K_{A_{1}}, K_{A_{2}}}$ and $K_{B_{1}}, K_{B_{2}}$ as follows:

$$
\begin{aligned}
& K_{A_{1}}=e\left(R_{B}+H_{1}\left(I D_{B}, R_{B}\right) P_{p u b}+T_{B}, R_{C}+H_{1}\left(I D_{C}, R_{C}\right) P_{p u b}+T_{C}\right)^{a+D_{A}} \\
& K_{A_{2}}=e\left(P_{B}, P_{C}\right)^{x_{A}} \\
& K_{B_{1}}=e\left(R_{A}+H_{1}\left(I D_{A}, R_{A}\right) P_{p u b}+T_{A}, R_{C}+H_{1}\left(I D_{C}, R_{C}\right) P_{p u b}+T_{C}\right)^{b+D_{B}} \\
& K_{B_{2}}=e\left(P_{A}, P_{C}\right)^{x_{B}}
\end{aligned}
$$

(5) $A$ and $B$ verify the integrity of the message received from $C$. If $M A C_{K_{A} / / K_{\mathrm{R}}}\left(I D_{C}, T_{C}, P_{C}, R_{C}\right)=M A C_{K_{\mathrm{q}} / / K_{\mathrm{q}}}\left(I D_{C}, T_{C}, P_{C}, R_{C}\right)=M A C_{K_{\mathrm{B}_{1}} / / K_{R_{2}}}\left(I D_{C}, T_{C}, P_{C}, R_{C}\right)$ are true, then $A$ will send $\left\langle I_{A}, M A C_{K_{4} / / K_{12}}\left(I D_{A}, T_{A}, P_{A}, R_{A}\right)\right\rangle$ respectively to $B$ and $C$, and $B$ will send $\left\langle I D_{B}, M A C_{K_{B_{1}} / / K_{B_{2}}}\left(I D_{B}, T_{B}, P_{B}, R_{B}\right)\right\rangle$ respectively to $A$ and $C$, and the parties concerned will respectively verify the integrity of the messages.

(6) If participants $A, B$ and $C$ follow the rules of the protocol during message exchange, then $K_{A_{1}}=K_{B_{1}}=K_{C_{1}}, K_{A_{2}}=K_{B_{2}}=K_{C_{2}}$ are true, and shared key $K l$ can be obtained through calculation, wherein:

$$
\begin{aligned}
& K_{1}=e(P, P)^{\left(a+D_{A}\right)\left(b+D_{B}\right)\left(c+D_{C}\right)} \\
& K_{2}=e(P, P)^{x_{A} x_{B} \chi_{C}}
\end{aligned}
$$

In order to ensure that the attacker cannot obtain any information from the session key, the three parties $A, B$ and $C$ implement Hash treatment to obtain the final session key $S K$, namely $S_{K}=H_{2}\left(K_{1} / / K_{2} / / P_{A} / / P_{B} / / P_{C}\right)$.

\subsection{Non-certificate Three-party Key Negotiation Protocol for Multi-KGC}

Similarly, the users can also obtain the session key in three different KGC fields according to the non-certificate key negotiation protocol. KGC1, KGC2 and KGC3 respectively represent three different key generation centers with the same system parameter generation, and the master keys thereof are ${ }^{s_{1}, s_{2}, s_{3} \in Z_{q}^{*}}$, and the master public keys of corresponding $\mathrm{KGC}$ are respectively $P_{1}=s_{1} P, P_{2}=s_{2} P, P_{3}=s_{3} P . A, B$ and $C$ are 
respectively the users of $K G C 1, K G C 2$ 和 $K G C 3$, and according to the protocol for single KGC, we can know:

$$
\begin{aligned}
& K_{A_{1}}=e\left(R_{B}+H_{1}\left(I D_{B}, R_{B}\right) P_{2}+T_{B}, R_{C}+H_{1}\left(I D_{C}, R_{C}\right) P_{3}+T_{C}\right)^{a+D_{A}} \\
& K_{A_{2}}=e\left(P_{B}, P_{C}\right)^{x_{A}} \\
& K_{B_{1}}=e\left(R_{A}+H_{1}\left(I D_{A}, R_{A}\right) P_{1}+T_{A}, R_{C}+H_{1}\left(I D_{C}, R_{C}\right) P_{3}+T_{C}\right)^{b+D_{B}} \\
& K_{B_{2}}=e\left(P_{A}, P_{C}\right)^{x_{B}} \\
& K_{C_{1}}=e\left(R_{A}+H_{1}\left(I D_{A}, R_{A}\right) P_{1}+T_{A}, R_{B}+H_{1}\left(I D_{B}, R_{B}\right) P_{2}+T_{B}\right)^{c+D_{C}} \\
& K_{C_{2}}=e\left(P_{A}, P_{B}\right)^{x_{C}}
\end{aligned}
$$

The shared key finally obtained is as follows:

$$
\begin{aligned}
& K_{1}=e(P, P)^{\left(a+D_{A}\right)\left(b+D_{B}\right)\left(c+D_{C}\right)} \\
& K_{2}=e(P, P)^{x_{A} X_{B} C_{C}}
\end{aligned}
$$

\section{Security and Efficiency Analysis of New Protocol}

\subsection{Security Analysis}

(1) Known session key security

In this protocol, the opponent is assumed to know the shared key of a session, namely $K 1$ and $K 2$, but the exposure of the shared key is useless for calculating the shared key for future session. In each new session, the users randomly select temporary keys a, b and c, and the opponent does not know the values of temporary keys $a, b$ and $c$; if the opponent wants to know $a, b$ and $c$, he must find $a, b$ and $c$ according to $T_{A}=a P, T_{B}=b P, T_{C}=c P$, wherein such problem is a discrete logarithm difficulty problem. Therefore, the new protocol can meet the known session key security requirement.

(2) Forward security

The opponent is assumed to know the long-term private keys of users $A, B$ and $C$, namely $s k A, s k B$ and $s k C$, but the opponent cannot calculate the shared key for the previous session, because the calculation of the long-term key not only depends on longterm keys $s k A, s k B$ and $s k C$, but also depends on temporary keys $a, b$ and $c$. Therefore, the new protocol can meet the forward security requirements.

(3) Resistance to key exposure impersonation attack

Resistance to key exposure impersonation attack involves in two kinds of opponents. For the first kind of opponents, the opponent does not know partial private keys $D A, D B$ and $D C$ of three new users $A, B$ and $C$, but can replace the public key of the users, namely: the opponent can randomly select secret values $x_{A}^{\prime}, x_{B}^{\prime}, x_{C}^{\prime}$ of users $A, B$ and $C$. Since the shared key is composed of $K 1$ and $K 2$, and according to the calculation formulae thereof, we can know: the opponent can calculate $K 2$ but cannot calculate $K 1$, because the opponent does not know partial private keys $D A, D B$ and $D C$ of users $A, B$ and $C$. Therefore, the new protocol can resist to the first kind of opponents.

For the second kind of opponents, the opponent knows master key $s$ of the system, namely: the opponent can calculate partial private keys $D A, D B$ and $D C$ of three users $A$, $B$ and $C$, but cannot replace the public keys of users $A, B$ and $C$, namely: the opponent does not know secret values ${ }^{x_{A}, x_{B}, x_{C}}$ of users $A, B$ and $C$. According to the calculation formula for the shared key, we can know that the opponent can calculate $K 1$ but cannot calculate $K 2$, so the opponent cannot calculate the shared key. Therefore, the new protocol can resist to the attack of the second kind of opponents. 
According to the above analysis, the following conclusion can be obtained: the new protocol can resist to the key exposure impersonation attack.

(4) Unknown key sharing security

For attacking the unknown shared key, the opponent must obtain $K_{1}=e(P, P)^{\left(a+D_{A}\right)\left(b+D_{B}\right)\left(c+D_{C}\right)}$ and $K_{2}=e(P, P)^{x_{x} x_{B} x_{C}}$, but the opponent does not know partial private keys $D A, D B$ and $D C$ of users $A, B$ and $C$, and the parties concerned have also verified the integrity of the messages. Therefore, it is impossible for the opponent to attack the unknown shared key.

(5) Resistance to temporary key exposure

During the protocol running process, when the opponent obtains temporary keys $a, b$ and $c$ of users $A, B$ and $C$, there are two kinds of opponents. For the first kind of opponents, the opponent does not know partial private keys $D A, D B$ and $D C$ of users $A, B$ and $C$, but can replace the public key of the users, namely: the opponent can randomly select secret values ${ }^{x_{A}}, x_{B}^{\prime}, x_{C}^{\prime}$ of users $A, B$ and $C$. Since the shared key is composed of $K 1$ and $K 2$, and according to the calculation formulae thereof, we can know: the opponent can calculate $K 2$ but cannot calculate $K 1$, because the opponent does not know partial private keys $D A, D B$ and $D C$ of users $A, B$ and $C$. Therefore, the new protocol can resist to the first kind of opponents.

For the second kind of opponents, the opponent knows master key $s$ of the system, namely: the opponent can calculate partial private keys $D A, D B$ and $D C$ of three users $A$, $B$ and $C$, but cannot replace the public keys of users $A, B$ and $C$, namely: the opponent does not know secret values ${ }_{A}, x_{B}, x_{C}$ of users $A, B$ and $C$. According to the calculation formula for the shared key, we can know that the opponent can calculate $K 1$ but cannot calculate $K 2$, so the opponent cannot calculate the shared key. Therefore, the new protocol can resist to the attack of the second kind of opponents.

According to the above analysis, the following conclusion can be obtained: the new protocol can resist to the temporary key exposure impersonation attack.

(6) Key uncontrollability

The final shared key depends on long-term private keys $s k A, s k B$ and $s k C$ and temporary keys $a, b$ and $c$ of three parties A, B and C, rather than those of one party. Therefore, the new protocol can meet this security attribute ---- key uncontrollability.

\subsection{Performance Analysis}

For the performance analysis, we mainly compare the operation workloads of bilinear pairings operation, point multiplication operation, exponentiation operation and Hash operation. The comparison result of the protocol efficiency is as shown in Table 1.

Table 1. Protocol Operation Cost Comparison

\begin{tabular}{ccccc}
\hline Protocol & $\begin{array}{c}\text { Bilinear } \\
\text { Pairings }\end{array}$ & $\begin{array}{c}\text { Point } \\
\text { Multiplication }\end{array}$ & Exponentiation & Hash \\
\hline Al-Riyami & 3 & 3 & 1 & 2 \\
Al-Riyami Protocol & 3 & 2 & 1 & 2 \\
Protocol Proposed by Chen Jiaqi & 2 & 2 & 2 & 2 \\
New Protocol & 3 & 2 & 1 & 2 \\
\hline
\end{tabular}

According to Table 1, the new protocol only needs two bilinear pairings for finally calculating the session key, but other protocols need three bilinear pairings. Compared with point multiplication operation, exponentiation operation and Hash operation, the 
bilinear pairings operation needs much more time, so the new protocol has higher efficiency.

\section{Conclusion}

In allusion to multi-KGC, a new authenticated non-certificate three-party key negotiation protocol is proposed in this paper. The result of the security analysis of the protocol shows that this protocol can meet the following security attributes: known session security, forward security, resistance to key exposure impersonation attack, unknown key sharing security, resistance to temporary key exposure, key uncontrollability, etc. The result of the comparison with other two protocols shows that the new protocol only needs to bilinear pairings for finally calculating the session key, but other protocols all need three bilinear pairings. Since the bilinear pairings occupy a large proportion in the calculation complexity of the protocol, so the new protocol has higher operation efficiency and can better meet modern communication security requirements.

\section{Acknowledgement}

This work was financially supported by project of Humanities and Social Sciences Research Project of Ministry of Education, China (14YJC630154); ZheJiang Nature Science Foundation of China (LY13G030031); ZheJiang Philosophy \& Society Science Foundation of China (16NDJC094YB); NingBo Nature Science Foundation of China (2014A610174); NingBo Soft Science Foundation of China (2014A10033); Zhejiang Federation of Humanities and Social Science Circle Foundation, China (2014Z079);

\section{References}

[1] J. Hu and Z. Gao, "Modules identification in gene positive networks of hepatocellular carcinoma using Pearson agglomerative method and Pearson cohesion coupling modularity[J]", Journal of Applied Mathematics, (2012) .

[2] Y. Geng, J. Chen, R. Fu, G. Bao and K. Pahlavan, Enlighten Wearable Physiological Monitoring systems: On-Body RF Characteristics Based Human Motion Classification Using a Support Vector Machine, no. 99, pp. 1-16.

[3] Y. Liang*, "Satisfaction with Economic and Social Rights and Quality of Life in a Post-Disaster Zone in China: Evidence from Earthquake-Prone Sichuan", Disaster Medicine and Public Health Preparedness, vol. 9, no. 2, pp. 111-118.

[4] Y. Liang*, "Correlations between Health-Related Quality of Life and Interpersonal Trust: Comparisons between Two Generations of Chinese Rural-to-Urban Migrants", Social Indicators Research, vol. 123, no. 3, pp. 677-700.

[5] Y. Liang* and P. Lu, "Medical insurance policy organized by Chinese government and the health inequity of the elderly: longitudinal comparison based on effect of New Cooperative Medical Scheme on health of rural elderly in 22 provinces and cities", International Journal for Equity in Health, 13:37, 1-15. DOI:10.1186/1475 -9276-13-37, (2014).

[6] Y. Liang* and D. Zhu, "Subjective Well-Being of Chinese Landless Peasants in Relatively Developed Regions: Measurement Using PANAS and SWLS", Social Indicators Research, vol. 123, no. 3, pp. 817835.

[7] Y. Liang* and X. Wang, "Developing a new perspective to study the health of survivors of Sichuan earthquakes in China: a study on the effect of post-earthquake rescue policies on survivors' healthrelated quality of life", Health Research Policy and Systems, 11:41,1-12. DOI:10.1186/1478-4505-11$41 \backslash$

[8] J. Hu and Z. Gao, "Modules identification in gene positive networks of hepatocellular carcinoma using Pearson agglomerative method and Pearson cohesion coupling modularity[J]", Journal of Applied Mathematics, 2012 (2012).

[9] Y. Geng, J. Chen, R. Fu, G. Bao and K. Pahlavan, (2015). Enlighten Wearable Physiological Monitoring systems: On-Body RF Characteristics Based Human Motion Classification Using a Support Vector Machine. PP(99), 1-16. 


\begin{abstract}
Author
Lin Zhu, she received her M.S. degree in Business Management from Fort Hays State University in the U.S. In 2009 She graduated from Business School of Rowan University in New Jersey, U.S. She got her MBA degree specialized in Finance. She is currently a lecturer in the Business College of SIAS International University at Xin Zheng Henan. Her research interest is mainly in the area of ECommerce, Finance and Electrical Integration. She has published several research papers in scholarly journals in the above research areas and has participated in several books.
\end{abstract}

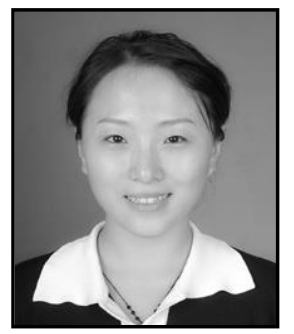

